\title{
Análise da prevalência dos transtornos psíquicos na região metropolitana do Recife
}

\author{
Analysis of the prevalence of psychic disorders \\ in the metropolitan region of Recife
}

\author{
Cíntia Paixão ${ }^{1}$ \\ Daniele $\mathrm{M}$ atias $^{1}$ \\ Izabel Alencar ${ }^{1}$ \\ Marina Nunes ${ }^{1}$ \\ Patrícia Sales ${ }^{1}$ \\ Paulo Henrique Altran Veiga ${ }^{2}$
}

\footnotetext{
${ }^{1}$ Curso de Fisioterapia, Universidade Católica de Pernambuco. Rua do Príncipe 526, Boa Vista. 50050-900 Recife PE. cintiapaixao18@hotmail.com

${ }^{2}$ Departamento de Biologia, Universidade Católica dePernambuco.
}

\begin{abstract}
M ental health is a state of well-being, in which the individual is aware of its capacities, capable of coping with the ordinary stress of everyday life, of working productively and of functioning in society. The purpose of the present work is a quantitative analysis of the prevalence of psychic di sorders, using references such as sex, age, kind of disorder and care deliver ed to the carriers. Social assistants of three psychiatric hospitals of the M etropolitan $\mathrm{Re}$ gion of Recife- thePsychiatric H ospital U lysses Pernambucano, the Nucleus for Psychosocial Care of Pernambuco (N APPE) and the H ospital Colony AIcides Codeceira - answered an adapted version of the Self Reporting Questionnaire (SRQ -20). The results showed a higher incidence of disorders in women in the age group between 41 and 60 years. Schizophrenia was the most frequently observed kind of mental disorder. Among the different treatments offered were drug therapy and individual and group therapy, the latter showing better results.
\end{abstract}

Key words Mental health, Psychic disorders, Schizophrenia
Resumo A saúdemental éum estado de bem-estar em que o indivíduo tem consciência das suas capacidades, écapaz de lidar com o estressenormal da vida, trabalhar produtivamente e contribuir para a sua comunidade. 0 presente trabalho tem como objetivo analisar quantitativamente a prevalência dos transtornos psíquicos, usando parâmetros referenciais como sexo, idade, tipos de distúrbios e assistência prestada aos portadores. Foi realizada uma abordagem quantitativa através de uma adaptação do questionário Self Reporting Questionnaire (SRQ - 20), em que foram entrevistadas assistentes sociais de três hospitais psiquiátricos da Região M etropolitana do Recife(RM R) - H ospital Psiquiátrico U lysses Pernambucano, N úcleo de Atenção Psicossocial de Pernambuco (NAPPE) e H ospital Colônia Aucides Codiceira. $O$ resultado mostrou uma maior incidência dostranstornos nas mulheres, entre a faixa etária de 41- 60 anos, destacando-se a esquizofrenia como 0 principal tipo de patologia dentre as encontradas. Entre os diversos tratamentos oferecidos, prevalecem as terapias medicamentosas, em grupo e individual, estas apresentando melhor resultado. Palavras-chave Saúde mental, Transtornos psíquicos, Esquizofrenia 


\section{Introdução}

A saúde mental, física e social são fios da vida estreitamenteentrelaçados e profundamenteinterdependentes, sendo indispensáveis ao bem-estar dos indivíduos em geral, das sociedades e dos países ${ }^{1,2}$.

Segundo a OMS, a saúde mental é um estado de bem-estar em que o indivíduo tem consciência das suas capacidades, é capaz de lidar com o estresse normal da vida, trabalhar produtivamentee contribuir para a sua comunidade. Ou seja, possuir o equilíbrio em todos os aspectos da vida física, social, mental e espiritual3,4.4.

Lamentavelmente, na maior parte do mundo, tanto a saúde quanto as perturbações mentais são negligenciadas e não recebem a mesma atenção e importância dadas à saúde física. Os esforços realizados para se divulgar e reduzir a repercussão negativa dos problemas de saúde mental são mal organizados e carecem de financiamento ${ }^{1}$.

A questão da assistência ao ser humano com transtornos mentais tem a sua trajetória marcada por processos de isolamento, segregação, exclusão e anulação do indivíduo enquanto portador dedireitos. Os grandes manicômios, construídos de prefe rência afastados dos centros urbanos, guardaram a imagem do "louco" e o estigma do medo longe dos cidadãos "normais" por um longo tempo $0^{3,5}$.

Essa forma de exercício da psiquiatria vem sendo questionada em todo o mundo, resultando em propostas de mudanças no sistema de atenção à saúde mental em vários países como Inglaterra, França, Estados Unidos e Itália. Através de caminhos diversos, todos foram unânimes em reconhecer a necessidade de ultrapassar o modelo centrado no hospital psiquiátrico e avançar no sentido do cuidado próximo às famílias e aos recursos socioculturais dos pacientes ${ }^{3}$.

0 contexto global em que se movem os projetos para mudança de enfoquee, conseqüentemente, de posturas, de propostas, de leis e de ações em saúdemental ébastante complexo e contraditório, como costumam ser os grandes processos sociais $s^{3}$. Tradicionalmente, considera-se que os transtornos mentais podem ser causados por agressão à integridade ou ao funcionamento do sistema nervoso central ou por influências psicológicas e sociais desfavoráveis ${ }^{6}$.

A saúde mental não é apenas o tratamento da doença, mas envolve também a prevenção, ao incorporar hábitos de vida saudáveis, bem como despertar a co-responsabilidade da comunidade, devendo envolver parcerias com outras secretarias e recursos comunitários ${ }^{3}$.

A atenção primária à saúde é um espaço pri- vilegiado que deve ser aproveitado para a promoção da saúde e prevenção de transtornos mentais. Os recursos disponíveis, como a cartilha e os folhetos do Programa de Saúde M ental, podem ser utilizados em ações de promoção da saúde e prevenção de transtornos mentais com os diversos grupos e integrados às atividades já desenvolvidas nas unidades ${ }^{4,7-9}$.

Para a construção de uma rede de atenção à saúde mental, fazem-se necessários recursos afetivos, sanitários, sociais, econômicos, culturais, religiosos e de lazer para potencializar as equipes de saúde nos esforços de cuidado e reabilitação psicossocial. Os CAPS (Centro de Atenção Psicossocial) devem estar articulados na rede de serviços de saúde e necessitam permanentemente de outras redes sociais e setores afins, em face à complexidade das demandas de inclusão daqueles que estão excluídos da sociedade por transtornos mentais ${ }^{10}$. As oficinas terapêuticas oferecidas pelo CAPS podem ser um elemento importante na construção de novos rumos para a atenção à saúde, integral e globalizante, ena perspectiva da totalidade, subjetividade e singularidade das pessoas.

Neste sentido, os terapeutas buscam um aparato teórico e técnico para o tratamento, procurando reinventar o cotidiano dos pacientes eatender aos interesses políticos eà saúde pública. Essas oficinas são atividades realizadas em grupo com a presença e orientação profissional. São realizados vários tipos de atividades, tendo em vista a maior integração social efamiliar, a manifestação de sentimentos eproblemas, o desenvolvimento de habilidades corporais, a realização de atividades produtivas, além do exercício coletivo da cidadania ${ }^{10,11}$.

0 presente trabalho tem como objetivo analisar quantitativamente a prevalência dos transtornos psíquicos de três instituições da região metropolitana de Recife (RM R), usando parâmetros referenciais como sexo, idade, a história de vida que predispõe à doença e a assistência prestada aos portadores.

\section{M etodologia}

Utilizou-seuma abordagem quantitativa através da adaptação do questionário Lüdke e André ${ }^{12}$ e Self Reporting Questionnaire (SRQ - 20), desenvolvido por $\mathrm{H}$ arding et al. ${ }^{13}$, em que foram empregadas técnicas de entrevistas semi-estruturada, entrevistando três assistentes sociais de três hospitais psiquiátricos da Região M etropolitana do Recife(RM R) - Hospital Psiquiátrico Ulysses Pernambucano, Núcleo de Atenção Psicossocial de Pernambuco 
(NAPPE) eH ospital Colônia Aucides Codiceira. Foi apresentada pelos pesquisadores e assinada pela direção dos hospitais estudados uma carta de anuência autorizando a realização do trabalho. Todas as assistentes sociais entrevistadas foram previamente contatadas e, após explicação dos objetivos do trabalho, assinaram um termo de consentimento livre e esclarecido, seguindo as conformidades dos princípios contidos na Declaração de H elsinque da Associação M édica M undial (1964, reformulada em 1975,1983, 1989, 1989, 1996 e 2000). Os questionários foram adaptados a fim de suprir informações em relação aos pacientes internos no momento da entrevista, e que eram assistidos pelas profissionais. Foram analisados indivíduos com transtornos mentais e os principais temas abordados foram: sexo, idade, tempo médio deinternação, transtornos psíquicos mais freqüentes e tipos de assistência prestada aos portadores.

\section{Resultados}

De acordo com as observações realizadas com assistentes sociais em seus locais de trabal hos contabilizou-seum total de 319 institucionalizados

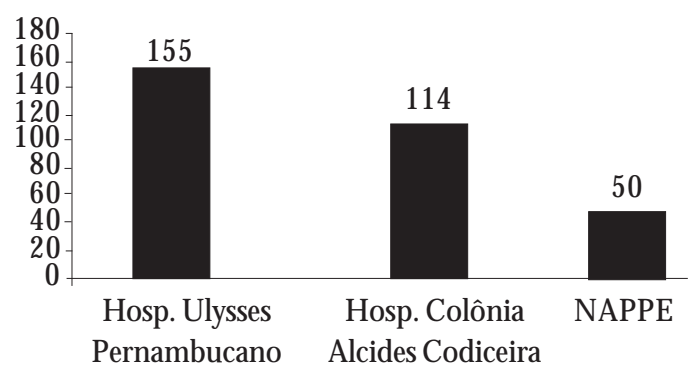

Figura 1. Número de internos.

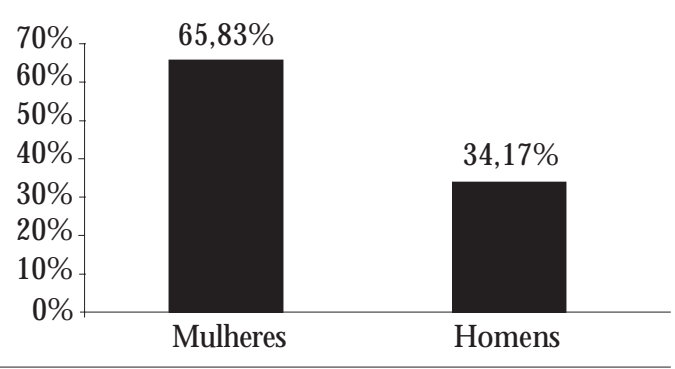

Figura 2. Sexo. distribuídos em três instituições psiquiátricas para analisar as variáveis citadas anteriormente.

Os dados em anexo mostram a distribuição dos internos por variáveis demográficas, sócioeconômicas e transtornos psíquicos presentes ( $\mathrm{Fi}$ guras 1 a 5).

Os resultados apontam um total de 319 internos das três instituições, entre eles observou-se o sexo feminino com $65,83 \%$ com altos índices de transtorno psíquico, em relação ao sexo masculino com 34,17\%. Em relação à idade pode-senotar que

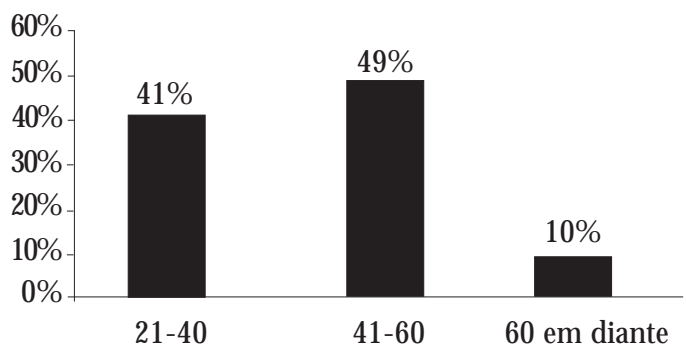

Figura 3. I dade.

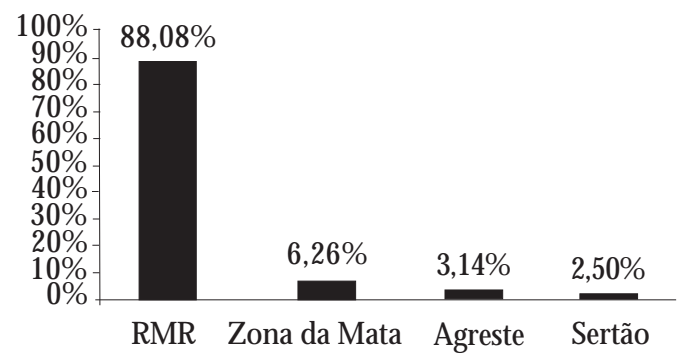

Figura 4. Regiões de origem.

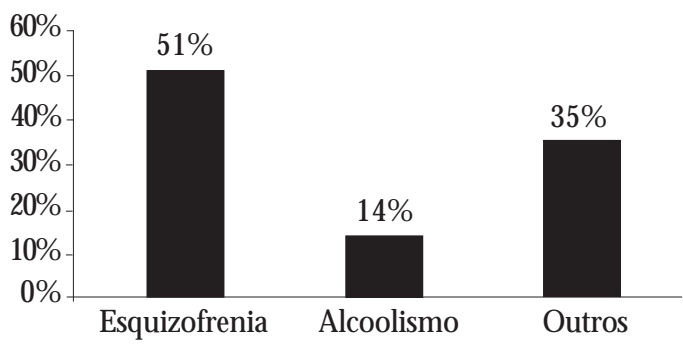

Figura 5. Tipos de transtornos psíquicos. 
a faixa etária na qual predomina as alterações psíquicas é entre 41 - 60 anos, com um total de $49 \%$, as outras faixas aparecem com $41 \%$ entre 21 - 40 anos, e apenas $10 \%$ com idade superior a 60 anos.

Dentre os institucionalizados, $88,08 \%$ são provenientes da região metropolitana do Recife (RM R), enquanto $6,26 \%$ vieram da Zona da M ata; em terceiro, aparece 0 agreste, com 3,14\%, e por último 0 sertão, com apenas 2,5\% dos internos. 0 transtorno psíquico predominante é a esquizofre nia com $51 \%$ e o alcoolismo com 14\%; os demais apresentam outros tipos de perturbações mentais.

\section{Discussão}

A esquizofrenia éum transtorno mental que atinge quase $1 \%$ da população mundial. Pode ser causada por diversos fatores que interagem, criando situações as quais podem ser favoráveis ou não ao aparecimento do transtorno ${ }^{14}$. Os fatores biológicos seriam aqueles ligados à genética ou àqueles que são devido a uma lesão ou anormalidade de estruturas cerebrais e deficiência em neurotransmissores. Os fatores psicossociais são aqueles ligados ao indivíduo, do ponto de vista psicológico e de sua interação com o seu ambiente social, tais como: ansiedade muito intensa, estado de estresse elevado, fobia social e situações sociais e emocionais intensas. Enfim, indivíduos com predisposição podem desenvolver a doença quando estimulados por fatores biológicos, ambientais ou emocionais ${ }^{15,16}$.

Pessoas esquizofrênicas têm como característica a perda de associações de idéias, alucinações, afeto embotado, riso imotivado ou inapropriado, avoliação [é isso mesmo?], alogia, delírios proe minentes, deterioração global do funcionamento, associações frouxas, desorganização da sintaxe, pensamento ilógico e, principalmente, pobre em acontecimentos ${ }^{14,16}$

Apesar da baixa incidência, por ser uma doença de longa duração, acumula-se, ao longo dos anos, um número considerável de pessoas portadoras desse transtorno, com diferentes graus de comprometimento edenecessidades. A intervenção no primeiro episódio do transtorno oferece uma oportunidade única no tratamento da esquizofrenia ${ }^{16}$.

Sabe-se que a demora na procura do tratamento tem uma influência fundamental no prognóstico do paciente, pois pode levar a uma ruptura significativa dos níveis psíquico, físico e da rede social do doente. 0 tempo de tratamento para obtenção da remissão do quadro agudo também aumenta à medida que se sucedem os episódios psicóticos ${ }^{8,16}$.

A interven ção adequada envolve o tratamento farmacológico, psicossocial e a inclusão da família. A intervenção psicossocial consiste no tratamento do paciente, baseado no envolvimento deste em atividades sociais e ocupacionais. Ela está sendo utilizada para diminuir o estigma da doença mental perante a sociedade ${ }^{16}$.

Em prol da cidadania das pessoas com transtornos mentais, a organização extra-hospitalar, do sistema assistencial e da prática interdisciplinar realiza um salto de qualidade frente a uma história repleta de tentativas de reforma, todas vinculadas a experiências européias ou norte - americanas bastante avançadas para cada época, mas com poucas críticas em relação à realidade socioeconômica e cultural brasileira. Dentre estes avanços, destacamse: ações de saúde mental em UBS (Unidades Básicas de Saúde), unidades ambulatoriais de atenção intensiva em saúde mental (hospitais-dia, CAPS), centros de convivência, unidade de saúde mental em hospital geral, isoladamente ou integrados em sistemas de referência ou contra-referência4.

O contexto da reforma psiquiátrica brasileira

A Portaria n 336/02 do Ministério da Saúde dispõe sobre o redirecionamento do modelo assistencial em saúde mental e os direitos das pessoas ditas portadoras de transtornos mentais. Discorre que esses cidadãos devem ser tratados, preferencialmente, em serviços comunitários "com humanidadee respeito eno interesseexclusivo de beneficiar a saúde, visando alcançar sua inserção na família, no trabalho e na comunidade" (Art. 2으, II). Segundo a Portaria no 336/2002 do Ministério da Saúde, os CAPS (Centros de Atenção Psicossocial - I, II, III) seriam os mais representativos desses serviços, que têm como prioridade "o atendimento de paciente com transtornos mentais severos e persistentes em sua área territorial, em regime de tratamento intensivo, semi-intensivo e não intensivo" (Art. 1ㅇ, 1) 1 .

Os resultados obtidos confirmam o que foi estudado anteriormente por Lima, que apresentou maior prevalência em mulheres (44,17\%); com idadeentre 41-60 anos (43,35\%). Deacordo com Bandeira, o tipo detranstorno psíquico predominante é o alcoolismo (40\%), contrariando os resultados obtidos nesse trabalho, no qual a esquizofrenia é mais comum nas institucionalizações estudadas.

As observações mostraram que a melhor forma de reintegrar o paciente na sociedade é através das terapias em grupos, como as oficinas de artes, capoeira, grupos de comunicação, estas sendo orientadas por assistente social, terapeutas ocupacionais, fisioterapeutas, entres outros. Estes profissi- 
onais também atuam com o objetivo de retornar o portador as suas AVDs e AIVDs, através de técnicas ocupacionais e orientações de higiene pessoal. Portanto, a fisioterapia não só pode ser empregada no setor terciário, estando responsável em reabilitar os portadores de transtornos que são seqüelados de AVC, entres outras patologias, mas também pode ser usada no setor primário, prevenindo contra doenças.

\section{Conclusão}

Conclui-se com o presente estudo que o transtorno psíquico predominante na região metropolitana de Recifeéa esquizofrenia, com uma incidência de $51 \%$ dos sujeitos analisados; o gênero feminino possui os mais altos índices de prevalência, com $65,83 \%$ dos casos; a faixa etária entre $41-60$ anos é aquela em que predominam as alterações psíquicas, e o convívio familiar ea integração social através de terapias em grupo éa forma mais rápida de retornar o portador à sociedade.

\section{Colaboradores}

C Paixão e D M atias realizaram o projeto inicial, escreveram 0 artigo e realizaram a interpretação dos dados e metodologia. I Alencar, $M$ Nunes e P Sales realizaram a captação e interpretação dos dados e metodologia. PHA Veiga realizou a revisão e orientação metodológica, revisão final eaprovação para publicação. 


\section{Referências}

1. Organização Mundial da Saúde. Mental health resources in the world: Initial results of Projet ATLAS. [periódico na Internet]. 2001 [acessado 2006 nov 15]; 29:[cerca de 20 p]. Disponível em: http://www.who. int/entity/whr/2001/en/whr01_ch1_po.pdf

2. Nutbeam D, Wise M, Bauman A, Harris E, Leeder S. Goals and targets for Australia's health in the year 2000 and beyond. Canberra: Australian Government Publishing Service; 1993.

3. Venetikides $\mathrm{CH}, \mathrm{M}$ aceno DM, Oliveira EA, Fabre LV Halboth NV, Simão M G. Protocolo integrado de saúde pública em Curitiba. 2002 [acessado 2006 nov 16]; [cerca de 111 p.]. Disponível em: http://www.curitiba. pr.gov.br/saude/sms/protocolos/mental.pdf

4. Nunes JM. Saúde mental. Rev Port Clin Geral 2006; 22(1):591-594.

5. Angermeyer $M C$, M atschinger $\mathrm{H}$. Public attitude towards psychiatric treatment. Acta Psychiatr Scand 1996; 94:326-336.

6. Barroso AGC, Abreu LM, Bezerra M AA, Ibiapina SLD, Brito HB. Transtornos mentais: o significado para os familiares. Rbps 2004; 17(3):99-108.

7. Martins LAN. Saúde mental nos profissionais de saúde. Rev. Bras. Med. Trab 2003; 1(1):56- 68.

8. Cretin S, Shortell SM, Keeler EB. An evaluation of collaborative interventions to improve chronic illness care: framework and study design. Evaluation Review 2004; 28:28-51.

9. Aarons G. Mental health provider attitudes toward adoption of evidence-based practice: Evidence-Based Practice Attitude Scale (EBPAS). M ental Health Services Researc 2004; 61-74.

10. Brasil. M inistério da Saúde. Saúde mental nos centros de atenção psicossocial. 1a ed. Brasília: Ministério da Saúde; 2004

11. Ribeiro M BS. Terapia ocupacional e saúde mental: construindo lugares de inclusão social. Interface (Botucatu) 2005; 9(17):425-431.

12. Breda MZ. O cuidado ao portador de transtorno psíquico na atenção básica de saúde. Cien Saude Colet 2001; 6(2):471-480.

13. Costa AG, Ludermir AB. Transtornos mentais comuns e apoio social: estudo em comunidade rural da Zona da M ata de Pernambuco, Brasil. Cad Saúde Pública 2005; 21(1):73-79.

14. Herz MI, Marder SR. Schizophrenia: Comprehensive Treatment and $M$ anagement. Philadelphia: Lippincott Williams \& Wilkins; 2002.

15. Ortiz LC. Esquizofrenia e transtorno bipolar. Notícias do M undo 2004; 56(1):22.

16. Giacon BCC. Primeiro episódio da esquizofrenia e assistência de enfermagem. Rev. Esc. Enferm USP 2006; 40(2):286-291.

17. Filho NGV, Nóbrega SM. A atenção psicossocial em saúde mental: contribuição teórica para o trabalho terapêutico em rede social. Revpsi 2004; 9(2):373-379.

Artigo apresentado em 19/06/2007

Aprovado em 04/12/2007

Versão final apresentada em 31/12/2007 